\title{
Relación entre la farmacología y la teoría de Florence Nightingale
}

\author{
Yoel. López Gamboa \\ yoel111975@gmail.com \\ https://orcid.org/0000-0002-9596-443X \\ Ciudad de Guayaquil. Provincia Guayas. Ecuador \\ Yoeldis Gamboa Pellicier. \\ gamboa41@gmail.com \\ Ciudad de Guayaquil. Provincia Guayas. Ecuador \\ Yanet Rodríguez Cantillo \\ alfrelia@gmail.com \\ https://orcid.org/0000-0001-5028-9576 \\ . Ciudad de Guayaquil. Provincia Guayas. Ecuador \\ Universidad Metropolitana de Ecuador.
}

\section{RESUMEN}

A mediados del siglo XIX es fundada la enfermería como profesión por parte de Florence Nightingale, aunque no es hasta la segunda mitad del siglo XX que verdaderamente se logran avances significativos en el desarrollo de la profesión. Con el objetivo de establecer la relación que existe entre la teoría de Florence Nightingale y la Farmacología se realizó el presente trabajo. Se hizo una revisión bibliográfica del estado actual del tema en las bases de datos Google académico, SciELO, Dialnet, y PubMed, con las palabras claves: Cronobiología, Cronofarmacología, Ritmos biológicos y Cronofarmocinética. En esta revisión se evidenció que existe una asociación directa entre el horario del día en que se suministran los fármacos, y los efectos farmacológicos, sobre todo por modificaciones en la variabilidad farmacocinética de los mismos, factores que también influyen en la seguridad de estos, y en la farmacodinamia. Los procesos fisiológicos que ocurren en nuestro organismo siguen el curso de ritmos biológicos, por lo que se deben tomar en consideración en la farmacoterapia con la finalidad de garantizar mayor eficacia terapéutica y mayor seguridad. Los elementos descritos nos permiten afirmar la relación entre la naturaleza y el hombre, según lo postulado por Nightingale, y la farmacología.

Palabras claves: cronobiología; cronofarmacología; ritmos biológicos; cronofarmocinética 


\title{
Relationship between pharmacology and Florence Nightingale's theory
}

\begin{abstract}
In the mid-nineteenth century, nursing as a profession was founded by Florence Nightingale, although it was not until the second half of the twentieth century that significant advances were truly made in the development of the profession. With the aim of establishing the relationship that exists Between Florence Nightingale's theory and Pharmacology, the present work was carried out. A bibliographic review of the current state of the subject was made in the Google academic, SciELO, Dialnet, and PubMed databases, with the keywords: Chronobiology, Chronopharmacology, Biological rhythms and Chronopharmokinetics. In this review, it was evidenced that there is a direct association between the time of day in which the drugs are administered, and the pharmacological effects, especially due to changes in their pharmacokinetic variability, factors that also influence their safety, and in pharmacodynamics. The physiological processes that occur in our body follow the course of biological rhythms, so they must be taken into consideration in pharmacotherapy in order to guarantee greater therapeutic efficacy and greater safety. The elements described allow us to affirm the relationship between nature and man, as postulated by Nightingale, and pharmacology.
\end{abstract}

Keywords: chronobiology; chronopharmacology; biological rhythms; chronopharmokinetics

Artículo recibido: 10 Agosto. 2021 Aceptado para publicación: 07. Setiembre. 2021 Correspondencia: yoel111975@gmail.com Conflictos de Interés: Ninguna que declarar 


\section{INTRODUCCIÓN}

A mediados del siglo XIX es fundada la enfermería como profesión por parte de Florence Nightingale, aunque no es hasta la segunda mitad del siglo XX que verdaderamente se logran avances significativos en el desarrollo de la profesión, y adquisición de competencias por parte del personal de enfermería, gracias a los avances teóricos y prácticos que se alcanzan en esta fecha. La milenaria práctica del cuidado a partir de este período inició a encontrar justificación teórica mediante la observación, la descripción, explicación, predicción y control de los fenómenos de su campo de actuación, especialmente el cuidado de los individuos, la familia y la comunidad. (NaranjoHernández et al., 2020)

La teoría unida a la práctica ha logrado fundamentar las acciones de enfermería como ciencia,que anteriormente solo era limitada al cumplimiento de órdenes médicas. Esta profesión no solo se dedica al ejercicio de la actividad práctica del cuidado de la salud, sino también a la educación e investigación asociadas a la solución de problemas emanados del ejercicio de la profesión como tal. La teoría ha aportado a la enfermería los análisis y razonamientos lógicos y el esclarecimiento de su actuar ético ante en los fenómenos estudiados. (Baeza et al., 2018)

Según Florence, toda mujer en algún momento debía servir de enfermera al cuidado de la salud de alguien. Definió como responsabilidad de la enfermera velar por la salud de otros en su nota de enfermería, donde también definió las líneas maestras sobre la práctica de la enfermería y dar consejos que permitieran a las mujeres pensar como enfermeras cuando tuvieran al cuidado la salud de los individuos. (Naranjo-Hernández et al., 2020) La teoría de Florence Nightingale hace hincapié fundamentalmente en las determinantes ambientales en el proceso salud-enfermedad, lo que ha sido motivo de asombro debido a que constituyó un pensamiento muy revolucionario para su tiempo donde existían otras teorías generalmente enunciadas por médicos, con énfasis en lo biológico. En dicho escenario Florence, definió la esencia de la profesión de enfermería que la hacía distintiva, y consistía en poner al paciente en las mejores condiciones para que la naturaleza actuara sobre él, definiendo además que la enfermería como profesión se basaba en el conocimiento de las personas y su entorno, lo que suponía una visión diferente vista hasta ese momento por los médicos para su ejercicio de la profesión. (Cano \& Carmen, 2004) 
Los postulados de Nightingale giran alrededor de tres elementos claves: la relación del paciente con su entorno, la relación de la enfermera con el paciente y la relación de la enfermera con el medio ambiente del paciente. (Cano \& Carmen, 2004) Dentro de estos tres elementos, según los expertos el menos explicado por Florence es la dimensión de la relación entre la enfermera y el paciente, no obstante, realizó varias observaciones en este sentido. (Martínez Bentancourth, 2018)

En este orden postuló y cito: "La enfermera debe explorar las preferencias de los pacientes acerca de los horarios de los tratamientos y cuidados, y sobre los contenidos y formas de alimentación, siempre que sea posible". (Cano \& Carmen, 2004)

En relación con esta última afirmación y poniendo en contexto la influencia que pueden tener los horarios del día en que son suministrados los medicamentos, en cuanto a la efectividad y seguridad de los mismos, con este trabajo se pretende realizar un acercamiento a una rama de la farmacología que estudia el citado fenómeno; la Cronofarmacología.

\section{MÉTODOS}

Se realizó una revisión bibliográfica del estado actual del tema en las bases de datos Google académico, SciELO, Dialnet, y PubMed, con las palabras claves: Cronobiología, Cronofarmacología, Ritmos biológicos, Cronofarmocinética, buscando algún nivel de asociación entre la teoría de Florence Nightingale y la Farmacología. La revisión se realizó en los idiomas inglés y español. Se utilizó bibliografía preferentemente de los últimos 5 años. No obstante, se tuvieron en cuenta publicaciones precedentes con elementos relevantes que pudieran argumentar el siguiente trabajo. Se encontraron 38 artículos con temáticas relacionadas, de los cuales 15 fueron tomados en consideración por abordar con mayor integralidad el tema en cuestión.

\section{RESULTADOS Y DISCUSIÓN}

Existe una asociación directa entre el horario del día en que se suministran los fármacos, y los efectos farmacológicos, sobre todo por modificaciones en la variabilidad farmacocinética de los mismos, factores que también influyen en la seguridad de estos, y en la farmacodinamia. Los ritmos biológicos afectan entre otros factores el metabolismo y la eliminación de los fármacos; en el metabolismo fundamentalmente porque las enzimas hepáticas con funciones metabólicas son sensibles a oscilaciones circadianas lo que influye directamente en los efectos farmacológicos y también en los efectos tóxicos 
(Molina Cabrera, 2015), (Zhao et al., 2020)

Los diferentes estudios científicos realizados en el campo de la cronobiología han demostrado elocuentemente la influencia que tienen los diferentes ritmos biológicos en las funciones vitales de los organismos vivos. Los ritmos biológicos se relacionan con determinada liberación hormonal en diferentes horarios del día que condicionan el comportamiento de la fisiología humana, que a su vez influye en que el organismo en determinados horarios del día presente una mejor asimilación de los tratamientos farmacológicos. (Molina-Rodríguez \& Akle-Álvarez, 2016)

La Cronofarmacología es un área de estudio relativamente nueva, que ha modificado la manera de visualizar algunos postulados que habían sido dado por sentados en cuanto a la efectividad de las terapias farmacológicas, su basamento estriba en los conceptos de la cronobiología, que estudian la influencia de los ritmos biológicos en las interacciones que existen a nivel celular y tisular con el entorno. (Castellanos et al., 2016)

Aun cuando los conceptos de cronobiología, y cronofarmacología no son muy nuevos, no se ha comprendido su importancia por parte del personal médico y otros actores de salud, razón por la cual de manera general no se tienen en cuenta a la hora de indicar una terapia farmacológica ,ni se ha integrado adecuadamente al comportamiento del proceso salud-enfermedad, por lo que no se tienen en cuenta los ritmos biológicos y se irrumpe en la biología de los seres humanos sin considerar las consecuencias que tendría para la salud, ni para la efectividad de la farmacoterapia.(Camprubí Andaluz, 2017)

Los procesos biológicos y de equilibrio que ocurren en el organismo humano cursan en el tiempo de manera cíclicos, pasando de manera gradual por varios estados hasta alcanzar sus valores máximos y mínimos, dentro de los que se pueden citar los siguientes: ciclo menstrual femenino, ciclo celular, ciclo sueño-vigilia, variaciones hormonales de cortisol, melatonina, adrenalina, serotonina, entre otras. Estas variaciones determinan que el organismo esté apto para determinadas funciones en los horarios donde las concentraciones de dichas hormonas presenten los mayores niveles de fluctuaciones. (Escagedo Cagigas, 2019)

En consonancia con lo explicado anteriormente, a continuación, se presentan algunos daños a la salud que pueden ser provocados por ruptura del equilibrio de algunos de los ritmos biológicos que ocurren en nuestro organismo. Considerando que los ritmos ultradianos o de alta frecuencia son aquellos que ocurren en tiempo menor a 20 horas, los 
ritmos infradianos o de baja frecuencia son los que ocurren en tiempo superior a 28 horas, y los circadianos los que ocurren entre 20 y 28horas.(Tamosiunas \& Toledo, 2010)

Tabla 1. Relación entre algunos ciclos biológicos, la fisiología y respuesta patológica del organismo

\begin{tabular}{|c|c|c|c|}
\hline No & Ritmos biológicos & Fisiología & Patología \\
\hline 1 & Circadianos & $\begin{array}{l}\text { Ciclo sueño-vigilia. } \\
\text { Temperatura corporal con } \\
\text { acrofase en la tarde. } \\
\text { Acrofase matinal (pico } \\
\text { máximo): catecolaminas, } \\
\text { cortisol, agregabilidad } \\
\text { plaquetaria }\end{array}$ & $\begin{array}{l}\text { Trastornos del sueño, } \\
\text { síndrome de cambio de uso } \\
\text { horario (Jetlag). Alteraciones } \\
\text { por trabajo en turnos rotativo. } \\
\text { Mayor incidencias de infarto } \\
\text { agudo de miocardio y } \\
\text { "Stroke" isquémico- } \\
\text { hemorrágico con mayor } \\
\text { incidencia en las mañanas }\end{array}$ \\
\hline 2 & & $\begin{array}{l}\text { Sistema renina- } \\
\text { angiotensa- aldosterona, } \\
\text { viscosidad sanguínea, } \\
\text { actividad plaquetaria, y } \\
\text { presión arterial, Acrofase } \\
\text { (pico máximo nocturno). } \\
\text { Secreción de ácido } \\
\text { clorhídrico, hormona } \\
\text { de crecimiento, melatonina } \\
\text { y } \\
\text { reactividad bronquial }\end{array}$ & $\begin{array}{l}\text { Mayor incidencia de úlceras } \\
\text { gas troduodenal en la noche. } \\
\text { Mayor incidencia de crisis de } \\
\text { broncoespasmo en la noche. } \\
\text { Edema agudo de pulmón. }\end{array}$ \\
\hline 3 & Ultradianos & $\begin{array}{l}\text { La frecuencia cardíaca, } \\
\text { respiratoria y la secreción } \\
\text { hormonal } \\
\text { pulsátil }\end{array}$ & $\begin{array}{l}\text { Arritmias cardíacas, polipnea } \\
\text { bradipnea }\end{array}$ \\
\hline 4 & Infradianos & El ciclo menstrual & $\begin{array}{l}\text { Síndrome disfórico } \\
\text { premenstrual }\end{array}$ \\
\hline
\end{tabular}

Fuente: Adaptación del artículo científico. La cronofarmacología: un nuevo aspecto a considerar en la variabilidad de la respuesta terapéutica.

La variabilidad de la respuesta terapéutica de los medicamentos depende de diferentes factores, y en cada individuo suele expresarse de una manera diferente, son mecanismos muy complejos y de diferentes naturaleza, comprende desde las propiedades fisicoquímica de los fármacos, pasando por la farmacoténica usada en la elaboración , 
hasta las diferencias etarias, sexuales, genética,polimedicación, hábitos de consumo de medicamentos y prescripción, así como influyen también la situación fisiopatológica de cada individuo. (Castellanos et al., 2016)

(Tamosiunas \& Toledo, 2010) plantea que los aspectos anteriormente citados son causas de modificaciones de la variabilidad farmacocinética y farmacodinámicas de los fármacos, lo que coincide con lo plateado por los autores Castellanos y Escobar. Además, refieren que la respuesta endógena del organismo frente a los diferentes ritmos biológicos, en lo que se refiere a respuestas bioquímicas, fisiológicas, hormonales y patológicas también modifican los parámetros farmacocinéticos y farmacodinámicos de los medicamentos y por ende modifican la respuesta farmacológica tras el consumo de determinados medicamentos, planteamiento en el que también coinciden los autores citados.

Según afirma (Tamosiunas \& Toledo, 2010), los estudios de cronofarmocinética encargados de evaluar la variación temporal de los diferentes parámetros farmacocinéticos dentro de los cuales destaca el tiempo de vida media y el tiempo en que se alcanza la concentración máxima del medicamento en el organismo; la variación rítmica de varios procesos biológicos tales como la secreción gastrointestinal, flujo sanguíneo, flujo hepático, filtración glomerular, afectan la absorción, distribución, metabolización y eliminación de los fármacos, por lo que no se puede garantizar que dichos parámetros farmacocinéticos sean iguales en los diferentes horarios del día.

Lo planteado por los autores anteriores se corrobora con los estudios farmacocinéticos realizados para explicar los factores que modifican los efectos farmacológicos de los medicamentos, ya sean los que dependen de los fármacos a través de sus propiedades fisicoquímicas y tecnológicas, así como los que dependen del enfermo. (Molina Cabrera, 2015), (Bicker et al., 2020)

Hay estudios que han demostrado variaciones circadianas a nivel hepático en los citocromos p4504A3 y N-acetiltransferasa las cuales actúan en el metabolismo de los fármacos tanto en la fase I como en la fase II, este hallazgo puede incrementar o disminuir el efecto del primer paso hepático en dependencia del horario del día en que se administren los medicamentos, lo que presupone una variación en los efectos farmacológicos de los mismos. (Tamosiunas \& Toledo, 2010). Es válido destacar que la cronofarmacocinética reviste vital importancia para aquellos medicamentos que presentan estrecho margen 
terapéutico tales como: la teofilina, litio, carbamazepina, ácido valpróico, y digoxina, entre otros. (Molina Cabrera, 2015)

Existen evidencias de varios fármacos que al suministrarlo en las mismas concentraciones y en similares condiciones ambientales, donde solo se ha modificado el horario del día muestra concentraciones plasmáticas diferentes. Por ejemplo, en un estudio realizado por, Orlando y col en Uruguay encontraron diferentes concentraciones de digoxina al darla en las condiciones explicadas con anterioridad, solo vario en que se suministró por la mañana en un caso y en el otro por la noche, y se evidenció que la concentración máxima del fármaco fue mayor en la noche que por la mañana.(Tamosiunas \& Toledo, 2010),(Alloway et al., 2020)

El hallazgo anterior pudiera ser explicado por los resultados obtenidos en otras investigaciones donde se explica que de manera general por las mañanas los procesos metabólicos se encuentran acelerados (Atienza Sánchez et al., 2016). Explicación similar pudiera darse al hecho de que otras investigaciones han comprobado que la teofilina cuando se administra por las noches tiene mejor seguridad y eficacia que al suministrase en otros horarios del día. (Tamosiunas \& Toledo, 2010)

Tomando en consideración la información que brinda la cronofarmacología se han establecidos pautas terapéuticas para diferentes patología, dentro de las cuales se pueden mencionar: tratamiento para la hipercolesterolemia, debido a que se conoce que en horarios nocturnos, entre la media noche y la madrugada se incrementa la síntesis endógena de colesterol, de suministra los Hipolipemiantes en la última hora de la tarde con el fin de alcanzar concentraciones máximas en los horarios de mayor síntesis. La hipertensión arterial suele incrementarse entre las seis de la mañana y hasta las doce del día, por lo que se recomienda suministrar la medicación durante la noche anterior de manera que se garantice concentración máxima en la hora de la mañana señalada; en la osteoartritis los síntomas son más intensos por la tarde, por lo que se debería suministra medicamentos en las mañanas. (Molina Cabrera, 2015), (Alloway et al., 2020)

Los glucocorticoides son un grupo de medicamentos que se usan con mucha frecuencia en el arsenal terapéutico actual sobre todo en enfermedades inflamatorias y del sistema inmunológico, dentro de las que destacan el asma bronquial, la artritis reumatoides, la enfermedad de Crohn, entre otras; se conoce que los ritmos biológicos que afectan a nuestro organismo influyen en la liberación de cortisol endógeno de nuestro organismo, 
el que constituye el primer glucocorticoide en los seres humano y si no se tiene en cuenta este elemento a la hora de administrar estos medicamentos puede verse afectada la regulación de cortisol por parte del hipotálamo e inhibirse la producción fisiológica de dicha sustancia. (Scherholz et al., 2019)

Existen muchas estrategias farmacológicas que tienen en consideración las variaciones de los ritmos circadianos, precisamente tomando en consideración las afectaciones que ellos provocan en las diferentes hormonas de nuestro organismo humano en los diferentes horarios del día. La desregulación endocrina producida por la liberación de cortisol puede conllevar al desarrollo de enfermedades cancerosas, metabólicas, depresión, insomnio, entre otras; en estos casos aplicar mecanismos terapéuticos basados en la cronofarmacología es vital para alcanzar efectos terapéuticos mas certeros que pueden influir en cambios biológicos, conductuales, bioquímicos y fisiológicos. (Ohdo et al., 2019), (Bicker et al., 2020)

\section{CONCLUSIONES}

- Los procesos fisiológicos que ocurren en nuestro organismo siguen el curso de ritmos biológicos, por lo que se deben tomar en consideración en la farmacoterapia con la finalidad de garantizar mayor eficacia terapéutica y mayor seguridad, lo que evidencia la relación entre el hombre y la naturaleza según la teoría de Florence Nightingale y la farmacología.

- La cronofarmacología juega un papel importante dentro de la industria farmacéutica, debido que permite ajustar el diseño de las formas farmacéuticas a las características de los diferentes ritmos biológicos, y con ello favorecer los efectos farmacológicos de los medicamentos.

- El conocimiento adecuado de los ritmos biológicos permite modificar el curso fisiopatológico de muchas enfermedades y con ello una mejor calidad de vida a los pacientes

\section{REFERENCIAS BIBLIOGRÁFICAS}

RAlloway, R. R., Trofe-Clark, J., Brennan, D. C., Kerr, J., Cohen, E. A., Meier-Kriesche, U., Stevens, D. R., Moten, M. A., \& Momper, J. D. (2020). Chronopharmacokinetics and Food Effects of Single-Dose LCP-Tacrolimus in Healthy Volunteers. Therapeutic Drug Monitoring, 42(5), 679-685. https://doi.org/10.1097/FTD.0000000000000773 
Atienza Sánchez, L., Celdrán Quecedo, R., Atienza Sánchez, L., \& Celdrán Quecedo, R. (2016). Impacto de las alteraciones del ritmo circadiano y del número de horas en vigilia-sueño sobre las hormonas y el metabolismo: Efectos en la salud [Info:eu-repo/semantics/bachelorThesis]. https://eprints.ucm.es/id/eprint/49036/

Baeza, A. Á., Palma, A. B., Cauich, E. M., Silva, A. S., Jurado, L. S., Báez, M. V. J., \& González, H. M. (2018). Automedicación en alumnos de licenciatura en Enfermería. Revista CONAMED, 22(3), 120-124.

Bicker, J., Alves, G., Falcão, A., \& Fortuna, A. (2020). Timing in drug absorption and disposition: The past, present, and future of chronopharmacokinetics. British Journal of Pharmacology, 177(10), 2215-2239. https://doi.org/10.1111/bph.15017

Camprubí Andaluz, I. (2017). Importancia de la cronoterapia oncológica en los cuidados de enfermería. http://repositori.upf.edu/handle/10230/28198

Cano, A., \& Carmen, M. del. (2004). Florence Nightingale, la primera gran teórica de enfermería. Revista Cubana de Enfermería, 20(3), 0-0.

Castellanos, M. Á., Escobar, C., Castellanos, M. Á., \& Escobar, C. (2016). De la cronobiología a la cronomedicina. Revista de la Facultad de Medicina (México), 59(2), 15-23.

Escagedo Cagigas, C. (2019). Papel de la cronomedicina en la prevención de patologías endocrinas. https://repositorio.unican.es/xmlui/handle/10902/16564

Molina Cabrera, R. (2015). La cronofarmacología en la respuesta farmacoterapéutica. Gaceta Médica Boliviana, 38(1), 28-29.

Molina-Rodríguez, M. A., \& Akle-Álvarez, V. (2016). Los ritmos circadianos en cáncer y la cronoterapia. Iatreia, 29(3), 301-311. https://doi.org/10.17533/udea.iatreia.v29n3a05

Naranjo-Hernández, Y., Álvarez-Rodríguez, R., Mirabal-Requena, J. C., AlvarezEscobar, B., Naranjo-Hernández, Y., Álvarez-Rodríguez, R., Mirabal-Requena, J. C., \& Alvarez-Escobar, B. (2020). Florence Nightingale, la primera enfermera investigadora. Revista Archivo Médico de Camagüey, 24(3). http://scielo.sld.cu/scielo.php?script=sci_abstract\&pid=S 102502552020000300014\&lng=es\&nrm=iso\&tlng=en 
Ohdo, S., Koyanagi, S., \& Matsunaga, N. (2019). Chronopharmacological strategies focused on chrono-drug discovery. Pharmacology \& Therapeutics, 202, 72-90. https://doi.org/10.1016/j.pharmthera.2019.05.018

Scherholz, M. L., Schlesinger, N., \& Androulakis, I. P. (2019). Chronopharmacology of glucocorticoids. Advanced Drug Delivery Reviews, 151-152, 245-261. https://doi.org/10.1016/j.addr.2019.02.004

Tamosiunas, G., \& Toledo, M. (2010). La cronofarmacología: Un nuevo aspecto a considerar en la variabilidad de la respuesta terapéutica. Archivos de Medicina Interna, 32(4), 65-69.

Zhao, M., Xing, H., Chen, M., Dong, D., \& Wu, B. (2020). Circadian clock-controlled drug metabolism and transport. Xenobiotica; the Fate of Foreign Compounds in Biological Systems, $50(5)$ 495-505. https://doi.org/10.1080/00498254.2019.1672120 